\title{
Acceso a servicios de salud: análisis de barreras y estrategias en el caso de Medellín, Colombia*
}

\section{Access to Health Services: Analysis of Barriers and Strategies in Medellín, Colombia}

\section{Acesso aos serviços de saúde: análise de barreiras e estratégias no caso de Medellín, Colômbia}

Fecha de recepción: 17-02-14 Fecha de aceptación: 17-03-14 Disponible en línea: 01-07-14 doi: 10.11144/Javeriana.rgyps13-27.assa

Cómo citar este artículo:

Restrepo-Zea JH, Silva-Maya C, Andrade-Rivas F, VH-Dover, R. Acceso a servicios de salud: análisis de barreras y estrategias en el caso de Medellín, Colombia. Rev. Gerenc. Polít. Salud. 2014; 13(27): 242-265. http://dx.doi.org/10.11144/Javeriana.rgyps13-27.assa

\author{
Jairo Humberto Restrepo-Zea** \\ Constanza Silva-Maya*** \\ Federico Andrade-Rivas**** \\ Robert VH-Dover*****
}

\footnotetext{
Artículo de investigación derivado del proyecto Acceso a los servicios de salud en la ciudad de Medellín: caracterización y propuesta para el monitoreo y la vigilancia, cuya primera fase fue realizada entre mayo del 2013 y mayo del 2014. Financiación: Secretaría de Salud de Medellín y Universidad de Antioquia (Convenio de Asociación 4600047513 del 2013).

* Economista y magíster en Gobierno y Asuntos Públicos. Profesor titular del Departamento de Economía y coordinador del Grupo de Economía de la Salud (GEs), Facultad de Ciencias Económicas de la Universidad de Antioquia. Dirección: Calle 67 No. 53-108 Of. 13-410 Medellín, Colombia. Correo electrónico: jairo.restrepo@udea.edu.co

*** Enfermera y magíster en Desarrollo Educativo y Social, investigadora asociada del Grupo Recursos Estratégicos, Región y Dinámicas Socioambientales (RERDSA), Instituto de Estudios Regionales (INER) de la Universidad de Antioquia. Correo electrónico: constasilvamaya60@gmail.com

***** Magíster en Salud Pública, investigador asociado del Grupo RERDSA, Instituto de Estudios Regionales (INER) de la Universidad de Antioquia. Correo electrónico: federicoandrade@gmail.com.

****** Ph.D. en Folklor, profesor asociado del Departamento de Antropología, Facultad de Ciencias Sociales y Humanas y coordinador del Grupo RERDSA, Instituto de Estudios Regionales (INER) de la Universidad de Antioquia. Correo electrónico: rvhdover@gmail.com.
} 


\section{Resumen}

Contexto: el acceso a servicios de salud es un tema de creciente preocupación, en el caso colombiano ha ganado importancia frente al aseguramiento. Objetivo: construir un concepto de acceso e identificar barreras y estrategias, desde la perspectiva de actores en Medellín. Material y métodos: investigación cualitativa con énfasis en tres zonas de Medellín. Resultados: se identificaron similitudes y diferencias en la visión del acceso, las cuales permitieron construir el concepto. Las barreras y los facilitadores se atribuyen a factores de oferta y de demanda. La mayoría de las barreras se dan en el acceso real, durante la búsqueda y continuidad del tratamiento. Algunas características sociales de la población también son factores determinantes. Discusión y conclusiones: los actores recurren a estrategias para solventar las barreras de acceso. El análisis cualitativo del concepto de acceso, de las barreras y las facilidades permite identificar alternativas que pueden servir de insumo para políticas en salud.

Palabras clave: acceso a los servicios de salud; equidad en salud; barreras en el acceso; política de salud; análisis cualitativo; Colombia

\section{Abstract}

Context: The access to health services is an increasingly worrying topic in the research and public policies agendas. In the case of Colombia, this topic becomes more important and attains supremacy opposite to assurance. Objective: Building a concept of access and identifying barriers, as well as strategies and alternatives to overcome them from the perspective of the actors of the health system in Medellin. Materials and Methods: qualitative research, focused on three areas of Medellin. We used techniques such as semi-structured interviews; social cartography workshops; observation and result questionnaires of the services. Results: The vision on the access is heterogeneous between actors, although some similarities and differences were identified, that allowed us to build a proposal of the concept of access. The barriers and factors that facilitate access can be attributed to factors on the offer side as well as on the demand side. Most of the barriers happen during real access, during the search and continuity of the treatment. The social characteristics of the population, such as knowledge of the system and social and community support are also determinant factors of access. Discussion and Conclusions: All actors use strategies to solve the access barriers and are affected by them on different levels. The qualitative analysis of the concept of access, of barriers and facilities, allow the identification of alternatives that may serve as components for health policies.

Keywords: access to health services; health equity; access barriers; health policies; qualitative analysis; Colombia

\section{Resumo}

Contexto: $\mathrm{O}$ acesso aos serviços de saúde é tema de preocupação crescente nas agendas de pesquisa e as políticas públicas. No caso colombiano, a questão ganha importância e encontra uma supremacia frente à asseguração. Objetivo: construir um conceito de acesso e identificar barreiras, estratégias e alternativas para superá-las, desde a perspectiva dos atores do sistema de saúde na cidade de Medellín. Material e métodos: pesquisa qualitativa, com ênfase em três zonas de Medellín. Utilizaram-se técnicas como entrevistas semiestruturadas, oficinas de cartografia social, observação e questionários de saída dos serviços. Resultados: a visão sobre o acesso é heterogénea entre atores, embora foram identificadas similitudes e diferenças que permitiram construir uma proposta de conceito de acesso. As barreiras e fatores que facilitam o acesso são atribuíveis a fatores tanto do lado da oferta quanto do lado da demanda. A maioria das barreiras ocorrem no acesso real, durante a procura e continuidade do tratamento. Características sociais da população, como conhecimento do sistema e apoio social e comunitário, são também fatores determinantes do acesso. Discussão e conclusões: todos os atores recorrem a estratégias para superar as barreiras de acesso e são afetados por estas em níveis diferentes. A análise qualitativa do conceito de acesso, das barreiras e as facilidades permite identificar alternativas que podem servir de insumo para políticas em saúde.

Palavras-chave: acesso aos serviços de saúde; equidade em saúde; barreiras no acesso; política de saúde; análise qualitativo; Colômbia 


\section{Introducción}

El acceso a los servicios de salud, uno de los retos más importantes que tienen principalmente los sistemas de salud de los países de mediano y bajo ingreso, se entiende como la capacidad que desarrolla una persona o un grupo de personas para buscar y obtener atención médica (1). Además de esta concepción ampliamente aceptada, en la literatura se encuentran variadas y diversas definiciones del concepto de acceso. Para Andersen, se trata de la "capacidad de utilizar los servicios de salud cuando y donde sea necesario" (2); para Cromely y McLafferty el acceso es el "poder de disponer de los recursos del servicio de salud" (3); para Dixon-Woods et ál., la forma más útil de entender el acceso es a través del concepto de candidatura, o candidacy, que describe la elegibilidad de las personas para la asistencia sanitaria, determinada por ellas mismas y por los servicios de salud (4). Entre las aproximaciones al acceso se destaca el carácter multidimensional del concepto, pues este incluye cuestiones como disponibilidad, accesibilidad, acomodación y aceptabilidad (5), lo cual hace posible acercamientos multidisciplinarios y transversales desde varios campos o disciplinas del conocimiento, que pueden incluir la salud pública, la antropología, la psicología y la economía, entre otras.

De las definiciones del acceso se destaca, como un aspecto central para dar lugar a la realización de este, el hecho de contar con capacidad para emprender la búsqueda de los servicios y para resolver la necesidad o los deseos individuales o colectivos. Se destaca que la capacidad puede estar formada a partir de características propias del individuo, como la edad, el sexo, su estado de salud o la percepción sobre este; del sistema de prestación de servicios, como el portafolio, la disponibilidad de recursos, los horarios de atención; y del contexto en donde se desenvuelve la persona, como las facilidades de transporte, aspectos propios de la cultura o la convivencia (6). También se destaca en las definiciones la consideración del acceso como un proceso en el que se identifican momentos claves como la necesidad, la búsqueda de servicios, el inicio de la atención y la continuidad en el sistema de prestación para resolver la necesidad (1).

La preocupación mundial por el acceso se encuentra en la agenda mundial para el desarrollo, en discusión de manera simultánea con la evaluación de los Objetivos del Milenio que se habían trazado para 2015. En esta agenda se hace énfasis en el acceso efectivo en términos de garantizar prestaciones médicas y protección financiera a toda la población (7). Además de las declaraciones políticas y del compromiso que se espera de los gobiernos para hacer realidad la universalidad en el campo de la salud, el Informe sobre la salud en el mundo de 2013 muestra la importancia de contar con una agenda de investigación para apoyar los retos de la cobertura universal, de modo que la investigación genere resultados para la adopción de buenas políticas y para la innovación tanto a nivel tecnológico como a nivel social (8). Se espera así un relacionamiento entre Academia y tomadores de decisiones que permita brindar mejor información para orientar las políticas y para realizar una evaluación continua de estas.

En este contexto, Colombia ha significado una experiencia interesante pues en el país se busca que el acceso pueda realizarse mediante la cobertura del seguro de salud. Luego de más de veinte años de haberse iniciado el proceso de reforma, el país está cerca de la cobertura universal del seguro (9). Sin embargo, es creciente la preocupación sobre el acceso, sobre la equidad y sobre la calidad, así que la propia legislación y la jurisprudencia han hecho más explícito el propósito esencial de garantizar el acceso y han reconocido a la salud como un derecho humano fundamental $(10,11)$. 
Un punto culminante, que a su vez abre una nueva etapa en el desarrollo del sistema, lo constituye la Ley Estatutaria aprobada en 2013 para reglamentar este derecho (12). Estas preocupaciones, documentadas en estudios nacionales $(13,14)$, hacen parte de la agenda del Gobierno, y es así como en el plan de desarrollo nacional 2010-2014 se reconoce que la prestación de servicios ha avanzado, pero que existen retos en cuanto al acceso y a la calidad de la atención debido a que se presentan barreras que se concentran en la falta de dinero y en la percepción de mala calidad, razones por las cuales no se usan los servicios de salud. Además, en la propuesta oficial de reforma al sistema de salud presentada a comienzos de 2013, y que finalmente no surtió su trámite en el Congreso (Proyecto de Ley ordinaria 210 de 2013), se reconoce que si bien la cobertura del sistema es casi universal, existen barreras que impiden que los usuarios puedan acceder y que estas se incrementan si los costos y la complejidad del tratamiento son mayores (15).

Entre las investigaciones sobre barreras y facilidades del acceso se destaca a nivel internacional la revisión sistemática de Hirmas Adauy et ál. (16). En esta revisión se identificaron las siguientes barreras más recurrentes, que suelen afectar en mayor medida a personas de menor nivel socioeconómico: 1 ) costo de medicamentos, consultas médicas y exámenes; 2) temor o vergüenza al ser atendido en un servicio de salud; 3) desconfianza en los equipos de salud y en el tratamiento prescrito, y 4) estigma social, creencias y mitos. Por su parte, los facilitadores más frecuentes fueron: 1) contar con redes de apoyo social; 2) valor asignado a la atención de salud para reducir riesgos y complicaciones, 3) adaptación de los servicios al paciente y programas de manejo de la enfermedad. Asimismo, la revisión muestra que existen pocos análisis de barreras y facilidades desde la perspectiva de los actores del sistema.
En el caso colombiano, la revisión sistemática de Vargas et ál. (17) muestra que a pesar de contar con un número relativamente amplio de estudios, realmente pocos profundizan en factores de contexto o en la perspectiva de los actores sobre los aspectos que determinan el acceso. Los estudios indican la existencia de barreras al acceso, referidas a la población (cobertura del seguro, ingresos o educación) y a las características de los servicios (accesibilidad geográfica, asuntos administrativos o calidad).

Además de contar con estudios de carácter regional, tanto con enfoques cuantitativos como con enfoques cualitativos $(18,19)$, o con estudios de caso en localidades o referidos a poblaciones específicas (20-22), Vargas y Molina (13) estudiaron el caso de seis ciudades colombianas y evidenciaron como gran limitante para el acceso que el sistema dé prioridad a la rentabilidad y a los costos, por encima del derecho a la salud. Las barreras identificadas en este estudio se dividieron en cinco tipos: 1) administrativas, 2) geográficas, 3) normativas, 4) poca oferta y 5) contexto cultural, social, político y económico.

Asimismo, se analizaron las estrategias más recurrentes que utilizan los actores para solventar las barreras: 1) urgencias, 2) tutela, 3) acudir a funcionarios "puente", con buena disposición y contactos, 4) acompañamiento de líderes locales con conocimiento, 5) esfuerzo de profesionales de salud para ayudar, por ejemplo, al transporte de pacientes, 6) estrategias de IPS para aumentar oferta y extender horarios, y 7) atención privada. Aunque este estudio diferencia claramente el aseguramiento del acceso, no hace un análisis del concepto por parte de los actores del sistema, ni discrimina las limitantes del sistema teniendo en cuenta las etapas del proceso de acceso. 
Por otro lado, además de las barreras identificadas en varios sistemas de salud, Abadía y Oviedo (23) analizan el sistema colombiano desde el concepto de los itinerarios burocráticos y concluyen que el sistema de salud puede ser considerado en sí una barrera estructural para el acceso. En esta línea, el trabajo de Vargas et ál. (24) analiza distintas estrategias y controles administrativos que se han implementado en la lógica de la competencia entre las Empresas Promotoras de Salud (EPS). ${ }^{1}$ Este trabajo compara las perspectivas de los actores del sistema y ubica los momentos del acceso en los que se presentan las barreras.

Otro aporte importante para comprender las barreras al acceso en Colombia es el trabajo de García-Subirats et ál. (25), pues analiza las barreras en distintas etapas del acceso, incluyendo el momento en el cual las personas inician la búsqueda. Según este estudio, las principales barreras se dan tanto en el acceso inicial como en la continuación a solucionar los problemas de salud, y constituyen en su mayoría barreras de tipo geográfico, económico y las relacionadas con las autorizaciones por parte de las EPS.

En el caso de Medellín, de manera similar a como se ha dado en el país, se ha hecho explícita la visión que da primacía al concepto de acceso sobre el de aseguramiento. El plan municipal de desarrollo para 2012-2015 reconoce dificultades en el acceso, tanto para la población no afiliada como para los afiliados a la seguridad social, y plantea

1 Las Empresas Promotoras de Salud (EPS) son las entidades encargadas de afiliar a la población y de administrar los recursos dispuestos por el sistema (una proxi de la prima del seguro, denominada Unidad de Pago por Capitación (UPC)) para garantizar la prestación de los servicios (Plan Obligatorio de Salud (POS)). Así que estas entidades, que pueden ser públicas, privadas o mixtas, son las responsables del acceso de la población afiliada, para lo cual deben contratar la prestación de servicios o incluso pueden realizar una prestación directa con los límites que se establecen a la integración vertical. como objetivo "gestionar y supervisar el acceso a los servicios de salud por medio de la planeación, la coordinación de actores [...] y el seguimiento a la prestación de servicios, con el fin de que los ciudadanos obtengan servicios de salud con calidad y eficiencia" (26). Aunque también se tienen estudios referentes al caso específico de Medellín (2729), hacen falta ejercicios sistemáticos en los que se indague por barreras y facilidades en las distintas etapas del acceso y en todos los servicios, así como en los que se analice la perspectiva de los distintos actores respecto a esta problemática.

En este contexto de la investigación y de las políticas del sistema de salud, en donde predomina la preocupación sobre el acceso efectivo a los servicios de salud, se propuso caracterizar la problemática y sentar las bases de un modelo para el monitoreo y la vigilancia del acceso a los servicios de salud en la ciudad de Medellín. El problema de investigación estuvo delimitado por la caracterización del proceso de acceso en la ciudad - teniendo en cuenta los servicios de entrada al sistema y la continuidad en este-,la identificación y el análisis de barreras al acceso, las estrategias y alternativas que desarrollan o plantean los actores para resolver la problemática y la posibilidad de realizar monitoreo a este proceso mediante indicadores cualitativos y cuantitativos.

En este artículo se presentan los resultados de una primera fase de la investigación, concretamente lo relativo al trabajo cualitativo y a los siguientes objetivos específicos: generar un concepto de acceso a los servicios de salud para el contexto de la ciudad de Medellín; analizar la situación de accesibilidad — barreras y facilidades para el acceso-, e identificar alternativas para la solución de la problemática del acceso, consultando las estrategias que siguen y la visión que poseen los actores del sistema de salud en la ciudad. 


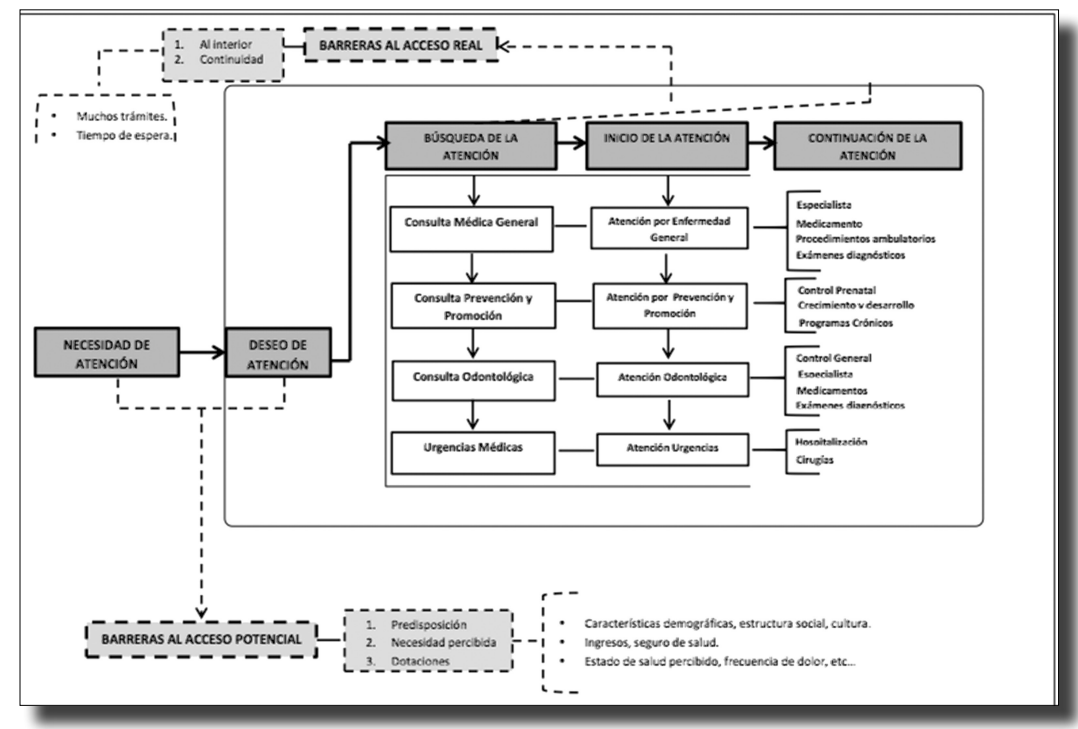

Figura 1. Dominios del acceso a los Servicios de salud para la Ciudad de Medellín

Fuente: adaptación de Frenk (1). Elaboración del Grupo de Economía de la Salud

La información suministrada permitirá, desde la mirada de los propios actores, mantener una línea de análisis y de investigación para mejorar la comprensión sobre el proceso y orientar políticas y acciones en pro de mejorar la situación. ${ }^{2}$

\section{Metodología y análisis de datos}

Se realizó una investigación de tipo analítico y explicativo. Analítica, al basarse en una conceptualización del proceso y en un análisis de las barreras y las estrategias que desarrollan los usuarios y los actores institucionales de la ciudad de Medellín para superarlas. Explicativa, pues buscaba establecer las causas o los factores que explican

2 El estudio también comprende un componente cuantitativo, por el cual se busca caracterizar la situación del acceso en la ciudad y contribuir a un análisis continuo que permita hacer comparaciones con la situación nacional y de otras ciudades. Es así como se hace una revisión de las fuentes de información sobre acceso potencial y real; además, se propone un conjunto de indicadores para el monitoreo. el acceso y las barreras y facilidades de este. El objeto de estudio fue analizado con una adaptación del esquema de Julio Frenk sobre los dominios del acceso (1), considerando su aplicación a cuatro servicios, sobre los cuales se enfatizaría en la indagación: consulta médica, consulta odontológica, programas de promoción y prevención y urgencias (ver figura 1). En el proceso de acceso se tuvieron en cuenta barreras al acceso potencial, en donde predominan las condiciones de las personas y su percepción sobre el sistema de prestación, y también al acceso real, en donde se pone a prueba el equilibrio o ajuste entre las necesidades y la oferta de servicios (30).

Con base en la recolección de información primaria, se consideraron dos niveles para el análisis. En primer lugar, se asumió la ciudad $^{3}$ como un todo y el proceso de ac-

3 Medellín es la capital del departamento de Antioquia y la segunda ciudad del país, por el número de habitantes y por su importancia económica. En ella habitan 2,4 millones de personas y junto con otros nueve municipios conforma el Área Metropolitana 
ceso en general, para lo cual se realizaron actividades, principalmente, con actores institucionales y líderes comunitarios.

El acercamiento a los actores se hizo con el convencimiento de que para comprender la perspectiva del acceso de quienes hacen parte del sistema, es indispensable aproximarse en forma diferente a cada uno de ellos y dimensionar así la complejidad de este. El análisis de estas perspectivas brinda información importante sobre el sistema y cómo los distintos actores construyen, entienden y se apropian de este. En cuanto a los actores institucionales (EPS, IPS, Secretaría de Salud y Personería de Medellín), teniendo en cuenta que estos actores tienen alta capacidad de agencia, se obtuvo información mediante entrevistas y talleres. Por su parte, dado el conocimiento del sistema y de la ruta para acceder a los servicios, por parte de líderes de usuarios y de asociaciones de usuarios y ONG que apoyan a pacientes con enfermedades de alto costo, con estos actores se realizaron entrevistas individuales y un taller.

En la perspectiva de ciudad, también se llevaron a cabo actividades para conocer la perspectiva de otros actores como los líderes de usuarios y de asociaciones de usuarios, de ONG que apoyan pacientes con enfermedades de alto costo para el sistema y de gran impacto para las personas. Las actividades con este

del Valle de Aburrá, en donde residen cerca de cuatro millones de personas, la mayor concentración demográfica y económica del departamento. La ciudad está organizada en dieciséis comunas en su área urbana, con 249 barrios y un $95 \%$ de la población, y cinco corregimientos en el área rural. La cobertura del Sistema de Seguridad Social en Salud supera el 90\%, un $64 \%$ de población cotizante y $28 \%$ de población subsidiada. Para la afiliación de esta población existen en la ciudad más de 10 eps del régimen contributivo, aunque cuatro concentran el $75 \%$ de los afiliados, y una del régimen subsidiado. En cuanto a la prestación de servicios, si bien cada eps organiza una red de prestadores para el acceso de su población, en el caso del régimen subsidiado la atención de los servicios básicos (primer nivel de atención y algunas especialidades, incluyendo hospitalización) se realiza a través de la red pública (Metrosalud). segundo grupo partían del supuesto de que sus miembros, por su perfil y liderazgo, están al día en el conocimiento del sistema, de la ruta para acceder a los servicios de salud, y entienden cómo buscar y prestar los servicios. En este caso se indagó desde su perspectiva, además de su concepto de acceso, por las barreras y estrategias para acceder a los servicios de salud empleadas por ellos y por sus miembros. Con ellos se realizaron talleres de cartografía del acceso, grupos focales, entrevistas individuales y grupales.

En segundo lugar, para ahondar en la situación del acceso en realidades más específicas y en donde fuera posible contar con información más detallada, se abordaron tres zonas o contextos ilustrativos de la ciudad, con los siguientes criterios para su selección: zona ilustrativa del área rural (corregimiento de San Cristóbal y parte del corregimiento de Palmitas); zona ilustrativa de población de estratos socioeconómicos medio-bajo y bajo, con importancia del régimen subsidiado de salud (comuna de Manrique), y zona ilustrativa de población de estratos socioeconómicos medio y medio-alto, con predominio del régimen contributivo de salud (comuna de Belén). En cada contexto, se abordaron los actores claves del sistema, especialmente desde las instituciones prestadoras de servicios y de las organizaciones comunitarias. Fue así como se seleccionó al menos una IPS ${ }^{4}$ según la población atendida y se convocó a su personal y a usuarios y líderes para realizar entrevistas, encuestas de salida de los servicios, talleres y cartografía.

4 En el caso del corregimiento de San Cristóbal fue posible recolectar información en el hospital de Metrosalud, que atiende a la población del régimen subsidiado, y en el centro de salud privado San Esteban, que atiende población del régimen contributivo; en Manrique, se contó con el hospital de Metrosalud y la ips de Comfama, que atiende población de la eps Sura, y en Belén también se contó con el hospital de Metrosalud y con una ips de Coomeva. 
Para la recolección de información se tomó el proceso de acceso desde la búsqueda de servicios hasta la continuidad en el sistema. Las técnicas empleadas, las cuales partieron de una explicación sobre los alcances de la investigación y de las consideraciones sobre consentimiento informado en los casos que era necesario, fueron:

- Talleres y jornadas de trabajo con actores institucionales y líderes comunitarios: se realizaron actividades con EPS e IPS, especialmente talleres, para construir el concepto de acceso y para conocer la manera como este se registra, se analiza y se gestiona. Además, luego de haber realizado convocatoria a todas las EPS con presencia en la ciudad, se hicieron entrevistas y búsqueda de información en Sura EPS, Coomeva, Nueva EPS y Savia Salud. Por otra parte, teniendo en cuenta la población atendida en las distintas zonas, se contó con la participación de las IPs de Metrosalud, Comfama y Coomeva. En cuanto a los líderes, se realizó un taller con treinta actores sociales (ligas de usuarios y comités de participación comunitaria) que cursaban un diplomado en control social organizado por la Alcaldía de Medellín.

- Entrevistas semiestructuradas: con duración promedio de treinta minutos, se buscaba profundizar en las experiencias de los actores en diferentes momentos del proceso de acceso.

- Talleres de sensibilización: actividades con actores para recolectar información mediante preguntas grupales y discusiones sobre la problemática del acceso.

- Grupos focales: entrevistas a grupos homogéneos de actores, como actividad complementaria en caso de necesitar validar o profundizar algunos temas encontrados en las demás actividades.

- Cuestionarios de salida: cuestionarios cortos para identificar la experiencia de los usuarios una vez que han utilizado los servicios de salud.

- Cartografía social y ruta de acceso: se realizaron talleres en los que los participantes dibujaron el recorrido para acceder a los servicios de salud, y además identificaron actores clave, agentes facilitadores, barreras y estrategias para el acceso.

- Observación: los investigadores llevaron a cabo observación en espacios relevantes para entender el acceso, como las salas de espera de urgencias o los sitios de entrega de medicamentos, donde se puede observar la experiencia de los usuarios frente al acceso.

Las preguntas empleadas en las técnicas con actores (tablas 1 y 2) buscaban, de manera general, conocer la manera como estos construyen, entienden y se apropian del sistema. Las preguntas se centraron en aspectos como: qué es el acceso, cuáles son los factores que lo determinan, cómo se ve el proceso en el entorno, cuál es la experiencia sobre el particular, qué dificultades se presentan, cómo se busca superar estas dificultades y qué tanto se conoce el sistema. Además, al querer conocer la perspectiva de los actores, era indispensable contar con un acercamiento diferente con cada uno de ellos y dimensionar así la complejidad del proceso, de modo que también se consultaba por la interacción que pudiera darse entre actores o la visión que se tiene de la participación de los demás en el proceso de acceso; por ejemplo, a los profesionales se les preguntó qué tanto entienden a los pacientes, y a los líderes si los usuarios conocen sus deberes y derechos. 
TABLA 1. Participantes en la investigación

(NÚMERo POR CONTEXTO Y ROL EN EL SISTEMA DE SALUd)

\begin{tabular}{|c|c|c|c|c|c|c|}
\hline \multirow[b]{2}{*}{ Régimen } & \multirow[b]{2}{*}{ Actores } & \multicolumn{3}{|c|}{ Contextos } & \multirow{2}{*}{$\begin{array}{c}\text { Ciudad en } \\
\text { general }\end{array}$} & \multirow{2}{*}{$\begin{array}{l}\text { Total por } \\
\text { régimen }\end{array}$} \\
\hline & & $\begin{array}{c}\text { San } \\
\text { Cristóbal }\end{array}$ & Belén & Manrique & & \\
\hline \multirow{5}{*}{ Subsidiado } & Líderes & 9 & 1 & 1 & \multirow{5}{*}{26} & \multirow{5}{*}{133} \\
\hline & Usuarios $\left({ }^{*}\right)$ & 20 & 20 & 26 & & \\
\hline & Profesionales & 8 & 6 & 5 & & \\
\hline & Administradores & 1 & 4 & 6 & & \\
\hline & Total & 38 & 31 & 38 & & \\
\hline \multirow{5}{*}{ Contributivo } & Usuarios & 8 & 13 & 16 & \multirow{4}{*}{24} & \multirow{4}{*}{77} \\
\hline & Profesionales & 3 & 3 & 4 & & \\
\hline & Administradores & 4 & 1 & 1 & & \\
\hline & Total & 15 & 17 & 21 & & \\
\hline & & & & & 50 & 210 \\
\hline
\end{tabular}

* Incluye entrevistas de larga duración y entrevistas cortas de salida.

Fuente: elaboración propia

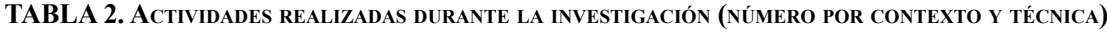

\begin{tabular}{|l|c|c|c|c|c|}
\hline \multirow{2}{*}{ Actividades } & \multicolumn{3}{|c|}{ Contextos } & Ciudad en \\
general & $\begin{array}{c}\text { San } \\
\text { Cristóbal }\end{array}$ & Belén & Manrique & Total \\
\hline Talleres (incluye cartografías) & 1 & 1 & 2 & 3 & 7 \\
\hline Entrevistas grupales & 3 & 2 & 1 & 2 & 8 \\
\hline Presentaciones & 4 & 1 & 1 & & 6 \\
\hline Cuestionarios de salida & 19 & 27 & 36 & & 82 \\
\hline Entrevistas & 34 & 21 & 21 & 8 & 84 \\
\hline Total & $\mathbf{6 1}$ & $\mathbf{5 2}$ & $\mathbf{6 1}$ & $\mathbf{1 3}$ & $\mathbf{1 8 7}$ \\
\hline
\end{tabular}

Fuente: elaboración propia

La información recolectada en el trabajo de campo se procesó utilizando el software de análisis de datos cualitativos NVivo 10. En este programa se incluyeron los archivos de audio e imágenes de las actividades, así como la transcripción parcial de aquellos elementos que se consideraron relacionados con la percepción del acceso. Durante el procesamiento de los datos, la información fue clasificada según los contextos y el tipo de actores, y se identificaron temas y conceptos relacionados con el acceso. Una vez ingresada la información, se configuraron unas primeras categorías para los análisis, se realizaron consultas y discusiones, y se procedió a la construcción —en Excel y en Word- de esquemas, cuadros de análisis y gráficas. Con toda la información organizada, se hizo una caracterización del acceso a los servicios de salud en cada uno de los contextos seleccionados, y luego el consolidado para la ciudad de Medellín, destacando diferencias entre contextos, si hubiere lugar a ello. 
Para la construcción del concepto de acceso, teniendo en cuenta la perspectiva de los actores, una vez identificados los temas principales e ilustrativos se realizaron tres propuestas de definición, resultantes de las definiciones dadas por cada uno de los actores, que fueron discutidas por el grupo de investigadores hasta llegar a una definición que posteriormente se validó con algunos de los participantes en la investigación. Para la validación, se construyó un cuestionario corto, en el cual se incluyó la propuesta del concepto de acceso para la ciudad — compartido mediante correo electrónico, llamadas telefónicas o jornadas de socialización de la investigación-, de modo que la persona manifestaba su acuerdo o desacuerdo con la definición, con la posibilidad de proponer cambios en la redacción. En el cuestionario utilizado para la validación también se indagó por algunos factores que facilitan $\mathrm{u}$ obstaculizan el acceso.

Finalmente, para la identificación de alternativas y propuestas para el mejoramiento del sistema de salud, se organizaron y consolidaron según los asuntos por mejorar planteados por cada uno de los actores, y se incluyeron aquellos que fueron identificados por los investigadores, a través de cada una de las actividades realizadas y que se consideraron que son necesarios para dar viabilidad a una propuesta de un modelo de monitoreo y vigilancia del acceso en la ciudad.

\section{Resultados}

\section{El concepto de acceso}

La visión sobre el acceso es bastante homogénea en el caso de las EPS, destacándose la idea de que se trata de la capacidad de recibir y solicitar servicios de salud por parte de los usuarios, así como de la responsabilidad del usuario de llevar a cabo el proceso de solicitar el servicio. También se refieren las EPS a las garantías de servicios que ofrecen las instituciones mediante la contratación o la instalación de una oferta adecuada. En cuanto a los determinantes para el acceso, aluden a la existencia y la eficiencia de los canales de comunicación e información de sus instituciones. Respecto a los obstáculos, además de mencionar la "sobreutilización de los servicios", predominan los que pueden atribuirse al sistema: "disponibilidad de profesionales o de servicios", "red insuficiente de la EPS", "oportunidad de citas", "barreras administrativas" y "capacidad de resolución”.

En el caso de las IPS, de parte de los directivos entrevistados, el acceso es entendido como la posibilidad de recibir atención médica en urgencias, citas médicas, programas de promoción o de prevención en las diferentes IPS, con independencia de la condición social y económica de la persona. En palabras recogidas por un grupo de participantes, el acceso es: "La posibilidad de ingresar a los servicios médicos a los que se está afiliado". Es así como se refieren al acceso en términos de posibilidad, facilidad y oportunidad, y entre los determinantes resaltan: la cercanía geográfica de los prestadores de servicios, el aseguramiento, la capacidad instalada, la calidad de los servicios, las cuotas moderadoras, la falta de información por parte del paciente, los aspectos culturales, la oportunidad en la atención, los trámites administrativos y la oferta de medicina especializada. Se resalta, además, que la medición del acceso se ve sobre todo en indicadores de oportunidad y de satisfacción. Las siguientes citas textuales ilustran la perspectiva de los administradores:

- "Es la oportunidad que tienen las personas de recibir atención en salud, la disponibilidad de poder venir y encontrar lo que necesiten". 
- "Tener la posibilidad expedita, libre, de obtener atención en salud en el momento en que se requiera. Tiene que ver con la accesibilidad, con no tener barreras geográficas, financieras, psicológicas o de lo que sean".

Por su parte, el personal administrativo de las IPS, como los facturadores o los encargados de la atención al usuario, entiende el acceso como el hecho de que el usuario asista a la institución y que allí se le preste el servicio, sin restricciones y con una calidad adecuada. Desde esta perspectiva, se da importancia a que el proceso sea fácil y oportuno. Aunque la mayoría de las referencias hechas por estos actores son respecto a servicios de asistencia a usuarios con necesidades inmediatas, se hicieron algunas referencias al papel de la promoción y la prevención:

- "Que la persona tenga un acceso fácil a la salud, a la atención inmediata en consulta médica, farmacia y laboratorio".

- $\quad$ "Por un lado está lo de conseguir una cita para la atención, y por el otro está lo de la promoción, porque no se tiene que estar enfermo para acceder".

Los profesionales incluidos en el estudio tienen una perspectiva similar del acceso a la de los administradores, así que este es visto como el proceso de atender a un usuario cuando lo solicita. Para ellos, el acceso es la satisfacción de una necesidad del usuario, la cual se debe brindar de manera oportuna, equitativa, fácil y rápida. Sin embargo, entre los médicos particulares se encontraron referencias a la calidad con la que es prestado el servicio, ya que estos incluyen en su discurso, de manera más frecuente, aspectos como el tiempo dedicado y la amabilidad con la cual es prestado el servicio, así como la posibilidad de aconsejar a los pacientes los tratamientos y los exámenes necesarios sin la presión de una institución que controle estos diagnósticos. Asimismo, los médicos particulares se expresaron sobre el derecho que tienen los usuarios a que se les prescriba el mejor tratamiento y que este sea acorde con los conocimientos adquiridos por los médicos, y no ceñido a las decisiones administrativas de las EPS. Algunas citas textuales ilustrativas:

- "Es la buena prestación del servicio de salud que se da a la comunidad. El calificativo de 'bueno' implica el fácil y rápido acceso a consultas, farmacia, remisiones".

- "Que las personas puedan consultar, enterarse a qué servicios tienen derecho y a qué servicios tienen facilidad para asistir a esos servicios".

En cuanto a los determinantes del acceso, los profesionales de la salud plantearon en su mayoría cuestiones similares. Sin embargo, según el régimen, dieron prioridad o se enfocaron más en unos determinantes que en otros. Para ambos regímenes, uno de los determinantes principales del acceso es la capacidad instalada de la institución y los distintos tipos de servicios ofrecidos. En el caso del régimen subsidiado, se hizo énfasis en la falta de conocimiento de los usuarios sobre los servicios de salud y en el uso deficiente de estos, lo cual fue tratado con menor énfasis por los profesionales que atienden población contributiva.

En cuanto a los líderes de usuarios y de organizaciones relacionadas con temas de la salud, estos suelen contar con un buen conocimiento acerca del funcionamiento del sistema de salud, así como de los derechos y deberes de los usuarios. De igual manera, varios de ellos cuentan con experiencia y han tenido contacto con usuarios que han padecido dificultades en el acceso. En el 
taller realizado con ligas de usuarios, las principales percepciones mencionadas fueron:

- "Es tener los mecanismos necesarios que permiten al ser humano la prevención y promoción de las enfermedades. A su vez es una ruta fundamental para que los ciudadanos tengan una vida digna y en paz".

- "Que los usuarios sean informados en sus derechos y deberes en cuanto al sistema de salud".

- "Ser muy cumplido, tener necesidad sí, y no hacer como trampas y ser correcto en el servicio, asistir a las citas y a todo lo mandado del médico del centro de salud".

Ahora bien, el concepto que tienen los líderes sobre el acceso puede enmarcarse en dos perspectivas. La primera muestra el acceso como el deber ser, donde se subraya la calidad de derecho y garantía para toda persona, y se hace énfasis en los aspectos de promoción y prevención que deben fomentar una vida digna y el trato humano a los pacientes. Por otro lado, el acceso es visto a partir de la experiencia y de las situaciones de las que los líderes tienen evidencias y relatos, así que se visibiliza como un "calvario" o como un "negocio".

Por último, aunque la perspectiva del acceso desde los usuarios es similar, en algunos puntos, a la de los administradores y a los profesionales de la salud, ellos hacen constante referencia a la manera en la cual el servicio es prestado y a la calidad de este. Algunas referencias textuales:

- "Que uno pueda contar con el servicio en el momento que uno lo necesite en una enfermedad o en un accidente".
- "Que presten un buen servicio. Que haya buen médico, que esté capacitado con todo lo que uno necesite".Énfasis en el tipo de atención que se da".

- “Tener acceso al centro de salud, tener buena atención, estar vinculada en ese centro de salud, o sea que yo tenga acceso, que yo esté afiliada a la EPS que corresponde a ese centro de salud”.

Por lo tanto, el usuario no solo reconoce la importancia de que el servicio sea prestado por el operador, sino que sea adecuado a las expectativas de calidad. En especial, busca que se le preste atención por un tiempo adecuado y que exista una relación de confianza con el médico y con la entidad en la cual está siendo atendido. Además, el determinante del acceso que se presentó de manera más constante fue el de la distancia geográfica. La manera en la que la barrera geográfica actúa depende del régimen del usuario, ya que varían los servicios a los que se puede acceder localmente o aquellos por los cuales se deben desplazar los usuarios a lugares como el centro de Medellín. De igual manera, el impacto que tiene el determinante geográfico sobre los usuarios también depende de su capacidad económica, ya que en el caso de poblaciones con fuertes limitaciones sociales y económicas el desplazamiento implica un esfuerzo muy alto.

Se resalta también que los usuarios reconocen falta de conocimiento de los derechos y deberes, así como del funcionamiento del sistema. Esta visión también es compartida por los profesionales de la salud, quienes ven en la falta de educación de distintos actores (incluyendo profesionales de la salud y administradores) una barrera para el acceso.

En resumen, basados en la perspectiva de los actores, se construyó un concepto de acceso que fue validado en la parte final de la investig- 
ación con varios de los participantes. Además, en un ejercicio de síntesis y de categorización, se definieron los determinantes que están presentes en las visiones de los distintos actores (tabla 3). El concepto es el siguiente:

El acceso a los servicios de salud en la ciudad de Medellín, es referido a la posibilidad de obtener, de una manera integral, los servicios de salud para satisfacer las necesidades de prevención y tratamiento de la enfermedad, y de conservación y mejoramiento de la salud; sin barreras, con oportunidad, equidad y calidad; garantizando el derecho fundamental a la salud y a una vida digna, y contando con la persona como corresponsable en el autocuidado y en el uso adecuado del sistema.

\section{Barreras, facilidades y estrategias para el acceso a los servicios de salud}

Luego de la aproximación al acceso, a continuación se presentan los hechos más destacados en cada uno de los contextos en donde se hizo el trabajo de campo: en la figura 2 las barreras al acceso referidas en los tres contextos, y más adelante las estrategias para afrontar o superar las barreras.

- San Cristóbal. En esta comunidad el determinante que predomina en el acceso es el geográfico, el cual se presenta tanto en el propio territorio, por la presencia de población rural, como ante la necesidad de desplazarse a la ciudad para lograr o continuar la atención, principalmente en el régimen contributivo. En cuanto al uso de los centros de salud, en el caso de la población del régimen subsidiado, existe la preferencia por dirigirse directamente a las unidades hospitalarias, ya sea en San Cristóbal o en otros lugares de Medellín. Además, se encuentra bastante establecido un sector privado de médicos generales, de odontología y de exámenes médicos, cuya atención es utilizada de manera recurrente y cuenta con buen prestigio entre los usuarios. Finalmente, hay

TABLA 3. FACTORES del ACCESO A LOS SERVICIOS DE SALUd (GRAdo DE IMPORTANCIA DAdo POR ACTORES)

\begin{tabular}{l} 
Conocimiento del sistema \\
Deberes y derechos, pasos por seguir, responsables, mecanismos de participación \\
\hline Características del entorno \\
Orden público, condiciones geográficas, redes sociales \\
\hline Capacidad del sistema para responder a las necesidades del usuario \\
Portafolio, disponibilidad y agenda de servicios, canales de comunicación \\
\hline Conocimiento sobre el usuario por parte de los actores el sistema \\
Reconocimiento de las necesidades, modelo de atención adecuado a la condición social \\
\hline Percepción o experiencias previas del usuario sobre el servicio \\
Experiencias previas, valoración del servicio y del recurso humano, expectativas \\
\hline Cercanía a los centros de atención \\
Distancia y facilidad de transporte \\
\hline Costos de los servicios \\
Copagos, servicios y medicamentos cubiertos, transporte \\
\hline Cultura \\
Estilo de vida, prácticas, creencias, saberes
\end{tabular}

Fuente: elaboración propia a partir de trabajo de campo 
grandes expectativas con la unidad hospitalaria de Metrosalud en construcción, las que tal vez no responden a los verdaderos alcances de la unidad, pues muchos creen que van a contar con nuevas especialidades y no que se trata de una ampliación de los mismos servicios.

- Manrique. El régimen contributivo solo está representado en la zona con la IPS Comfama, que atiende a los afiliados de la EPS Sura. Los afiliados a otras EPS deben desplazarse por fuera de la zona para poder iniciar la atención, y en general alguna población de ciertos sectores tiene dificultades de desplazamiento por razones de logística o por conflictos internos. La demanda de servicios de promoción y prevención tiene picos muy marcados, relacionados con las exigencias del programa "Familias en Acción". Existen quejas por parte de los profesionales de la salud y personal administrativo, por ejemplo respecto a los motivos por los cuales las madres acceden a estos servicios, pues que no siempre tienen una verdadera disposición de utilizar el servicio de manera adecuada, sino que se encuentran cumpliendo un requisito.

- Belén. La seguridad se identifica como un factor que limita a los usuarios para ir a los centros de salud o a la unidad hospitalaria más cercana a su lugar de vivienda. En especial, se mencionaron las "barreras invisibles" como un fenómeno importante en varios sectores. Por otra parte, en particular en el régimen subsidiado, continúa el uso de "fichos" (tiquete de turno), de manera paralela a la asignación de citas por teléfono y a las citas prioritarias. Los usuarios encuentran difícil la comunicación para solicitar servicios. El tamaño de la comuna, su diversidad y la cantidad de población, así como la dispersión de los centros de atención dificultan la generalización y la categorización del acceso. No fue fácil caracterizar al régimen contributivo, por dificultades para acceder a la información.

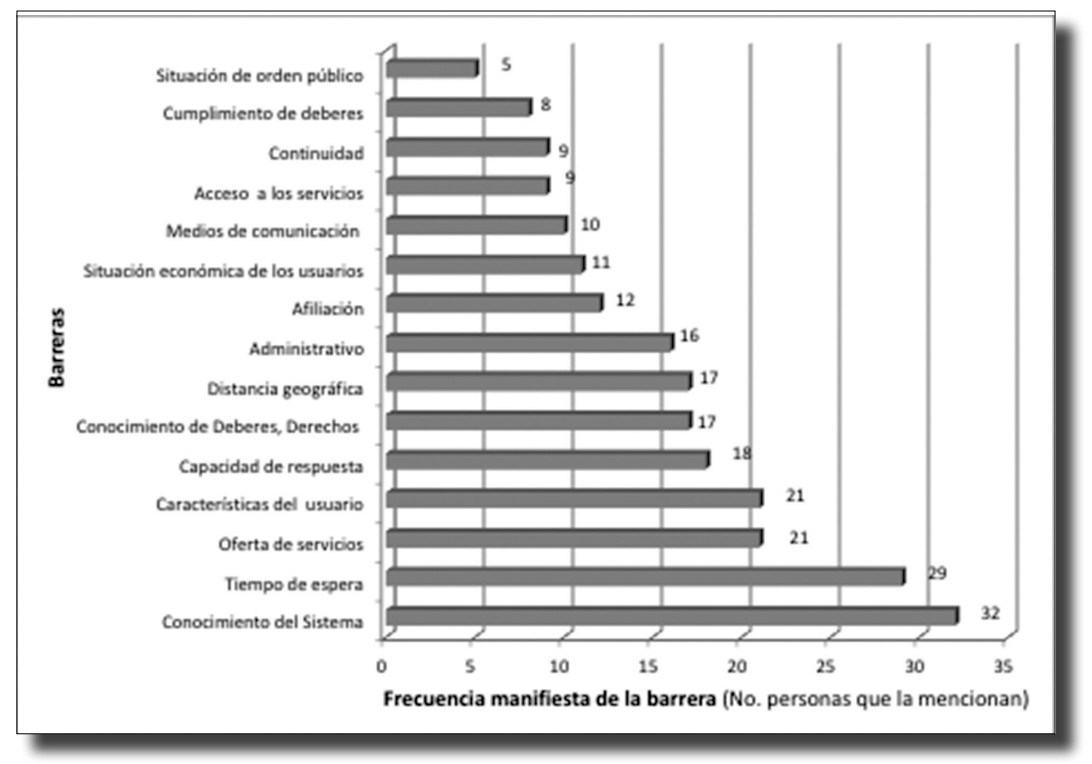

Figura 2. Medellín: Barreras al acceso a servicios de salud (Perspectiva de aCtores) 
Como respuesta a las barreras al acceso, los usuarios y los líderes de los diferentes contextos dan cuenta de varias estrategias para lograr acceder (ver tabla 4). Aunque no hay diferencias significativas entre los usuarios del régimen contributivo y los del subsidiado, existen algunos aspectos que solo aplican a alguno de los dos regímenes, como por ejemplo los que tienen relación con la encuesta del Sisben (subsidiado) y los asociados con la selección de EPS (contributivo). Por otra parte, en cuanto a las estrategias empleadas, no se encontraron diferencias entre los diferentes contextos; más bien, en conjunto, los hallazgos en los tres contextos constituyen una buena aproximación a lo que sucede en la ciudad. A continuación, los aspectos más destacados:

- La falta de conocimiento sobre derechos y deberes de los usuarios constituye una barrera para acceder a los servicios de salud. La falta de información lleva a que los usuarios no soliciten servicios sobre los cuales no reconocen el derecho o que lleven a cabo acciones sobre otros que congestionan el sistema. En el primero de los casos, los usuarios perciben que cuando demuestran conocimiento de sus derechos frente al personal administrativo y de atención, se presentan menores dificultades de acceso. Esta situación opera principalmente cuando los usuarios van a acceder a servicios de consulta general, odontología o urgencias, o cuando requieren acelerar un proceso que es responsabilidad directa de la institución prestadora o que esta puede tener gestión sobre ella.

- De igual manera, el conocimiento adecuado del sistema lleva a que se tenga una idea más acertada de en qué momento es vulnerado un derecho y se puedan llevar a cabo procesos legales. $\mathrm{Al}$ respecto, los usuarios acuden a líderes comunitarios o a ONG para buscar información o ayuda.
- La barrera geográfica fue mencionada con mayor énfasis en el caso de San Cristóbal, pero está presente en todos los contextos. Las dificultades se presentan en todos los servicios ofrecidos en el primer nivel y en los distintos puntos de la cadena de servicios del sistema. También se presenta en los procesos legales y de reclamos, que implica asistir a lugares específicos, como por ejemplo el centro de atención al usuario de la Secretaría de Salud.

- Para resolver la barrera geográfica, el usuario debe buscar maneras de financiar los desplazamientos para llegar al lugar donde se presta el servicio o se debe tramitar algún proceso. En muchas ocasiones, los usuarios recurren al apoyo de conocidos y familiares, ya sea para que proporcionen el transporte, la compañía o el préstamo del dinero necesario para el desplazamiento. Asimismo, hay usuarios que deciden pagar algunos exámenes de manera particular, ya que el desplazamiento al lugar donde el servicio se encuentra contratado por la EPS puede salir más costoso por los tiempos y por los gastos de desplazamiento.

- Es importante anotar que la distancia no solo es espacial, sino que también puede ser económica o social, debido a falta de recursos o al desconocimiento de cómo desenvolverse en ciertas zonas de la ciudad (aspecto crítico en el caso de la población rural de San Cristóbal). Los usuarios manifestaron que, en algunos casos, prefieren acceder al servicio por urgencias, incluso en situaciones no urgentes, ya que pueden acceder el mismo día u obtener una cita prioritaria. Igualmente, varios de los entrevistados manifestaron que al no poder acceder a una cita, o por los largos períodos de espera, deciden muchas veces acudir al 
farmaceuta o a un tegua para que les venda algo que les pueda ayudar con su problema de salud, o para sostenerse mientras pueden acceder a la cita médica o con el especialista.

- Acudir al servicio de urgencias, que se emplea para solventar barreras geográficas, es también una respuesta a barreras como las dificultades para comunicarse por vía telefónica con EPS o IPS para pedir citas, autorizaciones e información, así como al no poder acceder a medicamentos para dar continuidad a tratamientos vitales.

- Ante las dificultades en la continuidad, en cuanto a la entrega y al tipo de medicamentos formulados, los usuarios responden también con la compra particular y el "préstamo" de medicamentos entre usuarios con enfermedades similares.

- En cuanto a las dificultades para acceder a especialistas, los usuarios centran sus estrategias en acciones como los derechos de petición y las tutelas, así como en la búsqueda de apoyo en entidades como la Personería de Medellín y la Secretaría de Salud.

- Administradores y profesionales de la salud también reconocen barreras, tanto internas como externas al sistema de salud, para brindar la atención que quisieran a los pacientes. Igualmente, a veces llevan a cabo diferentes estrategias que agilizan y facilitan el acceso. La principal barrera es la dificultad en la continuidad del tratamiento, así que en ocasiones utilizan su red de conocidos en distintas instituciones para remitir a los pacientes que se encuentran en espera de ser ubicados por la EPs. Esta estrategia busca que se le asigne la oferta de especialidades a los casos considerados prioritarios (como mujeres gestantes o pacientes graves), o a usuarios que hayan despertado algún interés o preferencia en los profesionales o administradores de la salud. Adicionalmente, los profesionales de centros de primer nivel realizan ciertos procedimientos que no están autorizados, mientras al paciente se le adjudica una cama en el segundo nivel.

- Los administradores también resaltan actividades para expandir y hacer más efectiva la oferta, entre las cuales se resalta la ampliación de horarios de atención, asignar citas prioritarias a doctores que tuvieron inasistencia en citas programadas, hacer reuniones de personal en horarios de baja demanda, instalar un número telefónico para asignación de citas exclusivo para la unidad, llamar directamente a los usuarios para recordarles sus citas de control, y buscar descongestionar la demanda a través de ofrecer servicios en los puestos de salud locales (estrategia que se da en su gran mayoría en el régimen subsidiado).

\section{Alternativas y propuestas para mejorar el sistema desde la perspectiva de los actores}

Además de las estrategias planteadas para afrontar las barreras al acceso, la investigación dio cuenta de que los actores pueden plantear propuestas para resolver la problemática. A continuación, lo más destacado:

- Para los usuarios y los líderes, las soluciones están en el aumento de la cantidad y la calidad de la oferta y en la ampliación de horarios. Piden unos centros de salud con una mayor cantidad de servicios y la capacidad de llevar a cabo procedimientos que en la actualidad no realizan. 
- Los usuarios y los líderes del régimen contributivo piden que los servicios se provean más cerca al lugar de vivienda. También se dieron referencias a la importancia de fomentar los servicios de atención al hogar, sobre todo en casos de población vulnerable o con dificultad de ir a los centros de atención.

- De igual manera, usuarios y líderes consideran fundamental aumentar la cantidad y calidad del personal, ya que no consideran que este sea suficiente, particularmente en las especialidades.

- Los usuarios y los líderes también piden que los médicos tengan mayor libertad de formular, tiempo de atención para el usuario y un trato más humano. Este grupo de actores percibe como urgente la mejora en el sistema del reclamo de autorizaciones y la asignación de citas.

TABLA 4. MEdelLín: FaCILIDAdES y BaRRERAS PARA EL ACCESO A SERVICIOS DE SALUd

\begin{tabular}{|c|c|c|c|}
\hline & Atribuibles a & Facilidades & Barreras \\
\hline \multirow{19}{*}{ 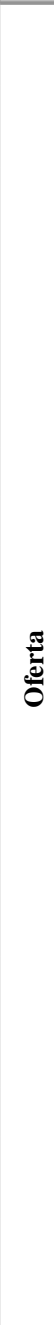 } & \multirow{4}{*}{ El Sistema } & La Constitución colombiana & $\begin{array}{l}\text { Falta de información para los cambios de } \\
\text { afiliación }\end{array}$ \\
\hline & & La normatividad en salud & $\begin{array}{l}\text { Desconfianza y falta de credibilidad en el } \\
\text { sistema y en los tratamientos autorizados }\end{array}$ \\
\hline & & $\begin{array}{l}\text { Existencia de mecanismos de } \\
\text { participación y de defensa de los } \\
\text { derechos en salud }\end{array}$ & $\begin{array}{l}\text { Existencia de dificultades cuando las } \\
\text { personas son de otros municipios }\end{array}$ \\
\hline & & $\begin{array}{l}\text { Redes de apoyo al usuario (Su- } \\
\text { persalud, Personería, centros de } \\
\text { atención a usuarios) }\end{array}$ & $\begin{array}{l}\text { Falta de actualización de datos del Sisben } \\
\text { y los puntajes asignados }\end{array}$ \\
\hline & \multirow{4}{*}{$\begin{array}{l}\text { La Adminis- } \\
\text { tradora (EPs) }\end{array}$} & \multirow{2}{*}{ Contratación con algunas IPS } & Demora y muchos trámites \\
\hline & & & Falta de orientación a los usuarios \\
\hline & & \multirow{2}{*}{ Demanda inducida } & Tiempos de espera \\
\hline & & & Limitaciones en los contratos con IPS \\
\hline & \multirow{11}{*}{$\begin{array}{l}\text { El prestador } \\
\text { (IPS) }\end{array}$} & $\begin{array}{l}\text { Ubicación geográfica IPs Régimen } \\
\text { subsidiado }\end{array}$ & Falta de asignación oportuna de citas \\
\hline & & \multirow{2}{*}{$\begin{array}{l}\text { Estrategias para inducir a pro- } \\
\text { gramas de promoción y prevención } \\
\text { (talleres) }\end{array}$} & Promoción de los programas y servicios \\
\hline & & & Calidad en la atención \\
\hline & & Consulta prioritaria & $\begin{array}{l}\text { Capacidad de respuesta a la demanda (re- } \\
\text { cursos humanos, insumos e infraestructura) }\end{array}$ \\
\hline & & $\begin{array}{l}\text { Buzón de comentarios, reclamos y } \\
\text { sugerencias }\end{array}$ & Conocimiento del sistema \\
\hline & & $\begin{array}{l}\text { Asesorías cortas sobre el sistema a } \\
\text { usuarios }\end{array}$ & Contacto con la entidad \\
\hline & & Aumento del personal médico & Limitaciones en los contratos con EPS \\
\hline & & Jornadas de promoción y prevención & Fragmentación de los servicios \\
\hline & & $\begin{array}{l}\text { Entrega de fórmulas para cuatro o } \\
\text { seis meses }\end{array}$ & Seguridad en los sitios de atención \\
\hline & & \multirow{2}{*}{$\begin{array}{l}\text { Estrategias para la asignación de } \\
\text { citas ("fichos", línea telefónica, } \\
\text { remisión) }\end{array}$} & Oferta de servicios \\
\hline & & & Tiempos de espera \\
\hline
\end{tabular}




\begin{tabular}{|c|c|c|c|}
\hline & Atribuibles a & Facilidades & Barreras \\
\hline \multirow{9}{*}{ 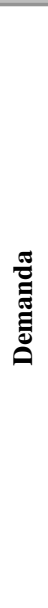 } & \multirow{5}{*}{ El usuario } & $\begin{array}{l}\text { Contar con redes de apoyo (funda- } \\
\text { ciones) }\end{array}$ & $\begin{array}{l}\text { Cumplimiento de requisitos para la } \\
\text { afiliación }\end{array}$ \\
\hline & & Asociaciones de usuarios & $\begin{array}{l}\text { Características del usuario (descuido, } \\
\text { despreocupación) }\end{array}$ \\
\hline & & $\begin{array}{l}\text { Acciones de defensa del derecho a } \\
\text { la salud (tutela, peticiones y acción } \\
\text { de cumplimiento) }\end{array}$ & Conocimiento de deberes y derechos \\
\hline & & \multirow{2}{*}{$\begin{array}{l}\text { Contactos o personas que laboran } \\
\text { en las IPS y que pueden facilitar } \\
\text { trámites }\end{array}$} & $\begin{array}{l}\text { Desconfianza y falta de credibilidad en el } \\
\text { sistema }\end{array}$ \\
\hline & & & Ingresos económicos \\
\hline & \multirow{4}{*}{ El contexto } & \multirow{4}{*}{$\begin{array}{l}\text { Cercanía geográfica de la red de } \\
\text { servicios }\end{array}$} & Seguridad para acceder a los servicios \\
\hline & & & Reclamación de derechos \\
\hline & & & Situación de orden público \\
\hline & & & Medios de transporte \\
\hline
\end{tabular}

Fuente: elaboración propia a partir de testimonios de actores participantes en la investigación

Existen muchas referencias a la necesidad de hacer más efectivos los medios para comunicarse con las entidades, lo cual va de la mano con la propuesta de usuarios y líderes de reducir la cantidad de trámites.

- Los líderes fueron enfáticos en la necesidad de que las comunidades se organicen y sean activas en la participación en las mejoras del sistema de salud. Manifestaron la dificultad de vincular a los jóvenes en mesas y grupos de salud.

- Los administradores y los profesionales de la salud enfocaron sus propuestas en mejorar la preparación de los usuarios para utilizar el sistema de salud. Consideran que estos actores no se comportan de una manera que permita que el sistema funcione de forma más adecuada y hacen especial énfasis en la falta de educación en deberes y derechos, así como en el funcionamiento del sistema y en los servicios que ofrece. Por lo tanto, proponen que se mejore la calidad y la cantidad de información.
- Existe una especial preocupación en estar creando una cultura asistencialista en los usuarios, lo cual causa mayores complicaciones en un futuro. A este respecto, el personal hace mención de sentir que en ocasiones se debe perseguir al usuario para que haga uso adecuado de los servicios, lo cual no debería ser la manera de funcionar del sistema. Asimismo, plantean que debe fortalecerse la educación en temas de autocuidado y de prevención, y ven con preocupación las dificultades que tienen los programas de promoción y prevención (por ejemplo, "Joven Sano") para lograr el impacto esperado, e incluso una asistencia aceptable.

- Tanto los profesionales de la salud como los administradores hicieron menos referencias que los usuarios a la capacidad instalada por parte de IPS. Sin embargo, también lo consideraron un tema por mejorar. Mencionaron la necesidad de mejorar los sistemas por los cuales los usuarios se comunican y solicitan servicios con la entidad, haciendo especial énfasis en la mejora del servicio telefónico. 
- $\quad$ Otro aspecto con relación a la oferta se refiere a la cantidad de personal. Los dos regímenes nombraron extensamente la necesidad de contar con más especialistas y servicios de alta complejidad, aunque las sugerencias respecto a la necesidad de aumentar la oferta en el primer nivel fueron más recurrentes por parte de los profesionales del régimen subsidiado.

- $\quad$ Las EPS, por su parte, plantean alternativas como: "capacitación”, "educación a la población y al personal de salud" y "uso racional por usuarios". En el orden institucional, se plantea: "planificación de la oferta y vigilancia”, "integración”, "mejorar tecnología”, "implementación P. y P.”, “mejorar atención de alta complejidad" y "agilizar trámites administrativos".

\section{Discusión}

Los hallazgos que revela esta investigación corroboran algunos aspectos tratados en la literatura o que han sido propuestos en otros trabajos. Se evidenciaron las barreras expuestas por Hirmas Adauy et ál. (16) para varios casos internacionales, así como las reportadas por Vargas y Molina (13) y García-Subirats et ál. (25) para el caso específico de Colombia. De igual manera, se encontró evidencia que apoya los resultados de Vargas et ál. (24) y de Abadía y Oviedo (23), donde se resalta de manera específica la importancia de las barreras administrativas y estructurales del sistema para comprender la problemática del acceso en Colombia.

En cuanto al momento en el cual se presentan la mayoría de las barreras, este estudio va en línea de lo encontrado por GarcíaSubirats et ál. (25), quienes sostienen que los problemas de acceso se presentan tanto en el acceso inicial como en la continuación.
Aunque no se presentó una mayor diferencia entre las barreras identificadas en este estudio y las presentadas en la literatura, es importante anotar que al analizar contextos específicos de la ciudad de Medellín, se encontró que el grado o prioridad que se le atribuye a cada barrera por parte de los actores depende de condiciones sociales, económicas, culturales y geográficas. Por lo tanto, es importante que al analizar la problemática del acceso, se tenga en cuenta que se pueden dar condiciones particulares que requieren políticas en salud específicas para situaciones particulares y que se deben tener en cuenta distintas escalas y poblaciones a la hora de estudiar el tema.

En el caso de los facilitadores del acceso, este estudio hace un especial énfasis en la importancia de las redes comunitarias y sociales con las que cuenta el usuario, así como el nivel educativo y el conocimiento específico que tiene este sobre el funcionamiento del sistema. Aunque Hirmas Adauy et ál. (16) mencionan de manera explícita las redes de apoyo social, esta revisión no encontró, como en el caso de este estudio, el conocimiento del sistema como uno de los facilitadores principales, tal vez por las particularidades del sistema colombiano. En el caso de Colombia, Vargas y Molina (13) identificaron facilitadores que pueden estar relacionados con el nivel educativo de los usuarios, así como las redes comunitarias y sociales de apoyo con las cuales cuentan. Sin embargo, estos autores no usan de manera explícita esta categoría de análisis, la cual se considera de gran importancia para promover políticas que fortalezcan el conocimiento de los usuarios del sistema, así como el acceso de estos a redes de apoyo.

Como resaltan Hirmas Adauy et ál. (16), son pocos los estudios que realizan un trabajo comparativo entre las percepciones de los distintos actores del sistema. El presente 
trabajo busca aportar en este aspecto, al complementar lo encontrado por Vargas et ál. (24), haciendo una comparación sobre algunas barreras no administrativas que han sido trabajadas con menor énfasis. De igual manera, el estudio aporta un ejercicio interesante al buscar complejizar la concepción que tienen los distintos actores sobre el concepto de acceso. Al momento de publicar no se conocen otros trabajos en Colombia que hayan llevado a cabo este ejercicio de manera sistemática, con miras a proponer un concepto local unificado de lo que se entiende por acceso. Se espera que este sea evaluado en Medellín y otros contextos y se reconozca la importancia de comprender lo que los distintos actores entienden por acceso a los servicios de salud.

Además de corroborar o matizar los resultados de otros estudios, este trabajo ofrece algunos aspectos que contribuyen a la investigación cualitativa en el campo del acceso a los servicios de salud. Un aporte es el conocer, comparar y sistematizar la percepción de los actores teniendo en cuenta el contexto social, económico y cultural en el cual se encuentran ubicados los servicios de salud. Otro aspecto, es el propósito de puntualizar en la percepción del concepto de acceso en servicios y buscar validarlo con los actores del sistema. De igual manera, el estudio da importancia al análisis de los distintos momentos del acceso, ya que esto permite ubicar dónde se presentan las mayores barreras y hace más específicas las alternativas que pueden implementarse.

\section{Conclusiones}

Hay una buena coincidencia entre la noción que tienen los actores acerca del acceso y la que ofrece la literatura, incluso en cuanto a los determinantes, aunque en general los actores se refieren al acceso real. No obstan- te, hay diferencias en las perspectivas que plantean los actores, con aspectos complementarios entre un grupo - conformado por usuarios, administradores y prestadoresque lo consideran como la posibilidad de recibir un servicio, y otro grupo - del que hacen parte principalmente líderes y profesionales de la salud - con una visión asociada al bienestar. El segundo grupo incluye en su discurso la importancia del autocuidado y de los estilos de vida, y al mismo tiempo concibe la salud como un derecho fundamental que no solo responde a los servicios prestados en los diferentes tipos de centros de atención. Aunque en ocasiones convergen el tipo de determinantes identificados, entre los que conciben la salud como un servicio y los que la ven como un estado de bienestar general, las soluciones y los determinantes que estos grupos identifican suelen diferir.

Aunque hay indicios sobre facilidades para acceder a servicios de atención básica, en particular a consulta médica general y odontológica, sin que al parecer se presenten diferencias significativas entre los regímenes de la seguridad social ni por estrato socioeconómico, según la percepción de la ciudadanía las diferencias se presentan en la calidad de los servicios, en la continuidad hacia servicios más complejos y, en general, en el goce del derecho a la salud. El mayor número de barreras encontradas en el trabajo cualitativo corresponden al acceso real, sin restar importancia a barreras del acceso potencial como la situación económica de los usuarios, las distancias geográficas y las características del contexto y de la población. Se destacan las barreras al acceso real por trabas administrativas, falta de infraestructura, falta de recursos profesionales y demora en las autorizaciones. Todo esto impacta la garantía del acceso a los servicios de salud como lo esperan las personas: oportuno, equitativo, de calidad y sin obstáculos. 
Con respecto a los momentos, según el esquema de Frenk, es en la búsqueda y en la continuidad en donde se dan mayores barreras. En la primera, se destacan las dificultades para comunicarse de los usuarios para solicitar citas. No se evidencian grandes barreras para las citas odontológicas, ni para acceder a los medicamentos y a exámenes de laboratorio que hacen parte del Pos. Las barreras se presentan en la continuidad, cuando los procedimientos, los exámenes diagnósticos y los medicamentos están por fuera del POS o se requieren citas con especialistas.

Las consultas por urgencias se ven afectadas por la cantidad de personas que solicitan este servicio, sin que en muchos casos sean emergencias médicas. Aunque hay claridad en los usuarios de cuál es el objeto del servicio, en ocasiones se trata de una estrategia para resolver las barreras en servicios ambulatorios o en los trámites para acceder a medicamentos o a procedimientos. Contrariamente a lo que sucede en urgencias, en donde la situación puede definirse en términos de una sobredemanda, no hay una conciencia sobre la importancia de la promoción y la prevención. En estos servicios no hay problemas de autorización, ni citas que solicitar, ya que se dan directamente cuando la persona asiste a control. La inducción de la demanda a estos servicios se realiza a través de otras atenciones, como cuando las personas asisten a consulta médica u odontológica.

Los programas que atienden enfermedades crónicas como la hipertensión y la diabetes, son los que mayor demanda tienen. Otros, como atención a la gestante y crecimiento y desarrollo, tienen acogida en la medida en que son requisitos para acceder a algunos subsidios del Estado, como los que otorga el programa Familias en Acción. En cambio, en programas como Adulto Sano y Joven Sano, no se encuentra una demanda importante.
Hay un evidente desconocimiento de las personas sobre cómo funciona el sistema de salud. El mayor conocimiento se concentra en los líderes, quienes son receptores de información, pero hace falta que esta llegue al resto de la comunidad. Igualmente, no se logra evidenciar un conocimiento claro de deberes y derechos en salud. Sin duda, hay una apropiación sobre los derechos, aunque no completa, pero frente a los deberes no se encuentra mucha claridad ni conciencia.

En cuanto a propuestas y estrategias para solventar barreras, los actores se pueden dividir en dos grupos: usuarios y líderes, y administradores y profesionales de la salud. Aunque existen algunas particularidades, en términos generales se encuentra congruencia entre los conceptos y los discursos analizados. Aunque ambos grupos de actores reconocen dificultades en su propio proceder, la mayoría de las sugerencias se hacen sobre aspectos de los cuales no son directamente responsables. Por ejemplo, los usuarios hacen un mayor énfasis en la necesidad de aumentar la capacidad instalada de servicios, mientras que en las IPS se enfatiza la necesidad de que los usuarios mejoren la manera en la que hacen uso de los servicios. Esto sin desconocer la necesidad de mejorar comportamientos propios como, por ejemplo, una mayor organización comunitaria por parte de los usuarios y una mejor disposición de servicio por parte del personal.

Es interesante que el conocimiento que tienen los usuarios del sistema actúe como un facilitador del acceso, reforzando la necesidad de capacitar a este grupo de actores. De igual manera, cabe resaltar la importancia de las redes sociales y comunitarias con las que cuenta el usuario, ya que estas le permiten acceder a distintas estrategias para solventar las barreras al acceso. En el caso de los actores institucionales de las EPS, estos, al igual que los usuarios, hacen un mayor énfasis en 
alternativas para mejorar el acceso direccionadas a mejoras en la oferta.

Se resalta el hecho de que todos los actores del sistema se ven afectados por las barreras de acceso. Aunque los usuarios son los que reciben los efectos, en detrimento directo a su salud, los administradores y profesionales perciben presiones del sistema que dificultan sus labores y generan frustración, por la tensión existente entre ejercer su labor y responder a las exigencias de una empresa. Con tal motivo, estos también despliegan estrategias, en ocasiones fuera de la norma, para posibilitar el acceso de los usuarios a los servicios de salud y poder poner en práctica sus conocimientos en pro de la salud.

Por último, una vez que se ha conocido la visión de los actores claves que hacen parte del sistema de salud, desde una perspectiva sociológica y de políticas públicas, se destaca la importancia de contar con la voz de dichos actores, muy especialmente de los usuarios, incluyendo el debate sobre reforma al sistema de salud. Es así como se encuentra esencial que para mejorar la situación del acceso, se desarrollen estrategias de comunicación y de diálogo con organizaciones y líderes comunitarios, pues ellos poseen información relevante acerca de la manera como se resuelve el acceso en sus comunidades. En el mismo sentido, para todos los usuarios deben fortalecerse los sistemas de atención mediante los cuales se pueden conocer y resolver peticiones, quejas, reclamos y solicitudes presentadas en cualquier momento en que se puedan presentar dificultades o sugerencias para mejorar el acceso.

En el caso de Medellín, el trabajo que sobre el particular lleva a cabo la Secretaría de Salud para brindar orientación y apoyo al ciudadano, a partir de las intervenciones que se realizan por medio del sitio web, de llamada telefónica o de asistencia a varios sitios de atención, puede verse como un sistema que reúne información para facilitar el acceso a los servicios de salud y acompañar al ciudadano en la resolución de las barreras que pudieran presentarse. Esto implica darle un mayor reconocimiento a la información y al equipo de trabajo que está a cargo de esta, y mejorar la gestión de la base de datos que se origina, de modo que sirva como insumo para el monitoreo y la vigilancia sobre el acceso.

Ahora bien, para contar con una mayor y mejor participación ciudadana, se requiere llevar a cabo labores de pedagogía para la comprensión y la apropiación del sistema de salud. Se hace necesario implementar un proceso educativo que favorezca la habilitación de la población para que sea sujeto y no objeto del sistema de salud, y para que logre desarrollar conocimientos, habilidades, destrezas y actitudes que le permitan conocer, comprender y apropiarse del sistema, logrando además ser ciudadanos corresponsables en el cuidado de su salud, y partícipes activos, vigilantes, dinamizadores y eficientes.

Para esto, se requiere una propuesta para realizar estrategias de capacitación que den cuenta del ser y del hacer en la prestación y en el uso de los servicios de salud, valorando los avances y reforzando los elementos técnicos y administrativos necesarios para dinamizar el proceso; propiciando experiencias que posibiliten el aprender-haciendo para el logro de aprendizajes significativos.

Se proponen dos tipos de actividades educativas: una orientada a la formación de usuarios, sobre la participación social en salud y el ejercicio de deberes y derechos, y otra para los funcionarios de IPS y EPS, encaminada a ofrecer herramientas al personal administrativo y asistencial de las IPS y EPS para mejorar sus interacciones comunicativas con los afiliados a través de la sensibilización con respecto a las condiciones socioeconó- 
micas y a las vivencias de los afiliados, y de la conciencia de sus propias experiencias; además sobre el sistema y sobre derechos y deberes de los usuarios.

\section{Agradecimientos}

Los autores agradecen a las personas e instituciones que colaboraron brindando información y aportando sus opiniones para que este estudio fuera posible. El trabajo contó con financiación de la Universidad de Antioquia y de la Secretaría de Salud de Medellín. De esta última, además, se contó con el aporte de los profesionales Lina María Ochoa Mejía, Fabián Ibarra Ruiz y Carlos Alberto Herrera Samudio. Asimismo, se agradece a la economista Diana Isabel Londoño y a los estudiantes Camilo José Liñán, Esteban Augusto Sánchez y Pablo Zabala, por su participación en la recolección y en el análisis de los datos.

\section{Referencias bibliográficas}

1. Frenk J. El concepto y medición de la accesibilidad. Salud Públ Méx. 1985; (27): 438-53.

2. Andersen R. Revisiting the Behavioral Model and Access to Medical Care: Does It Matter? J Health Soc Behav; 1995; 36 (1):1-10.

3. Cromley EK, McLafferty SL. GIS and Public Health. UK: The Guilford Press; 2002.

4. Dixon-Woods M, Cavers D, Agarwal S, Annandale E, Arthur A, Harvey J et ál. Conducting a Critical Interpretive Synthesis of the Literature on Access to Healthcare by Vulnerable Groups. BMc Med Res Methodol. 2006; 6 (35).

5. Graves A. A Model for Assessment of Potential Geographical Accessibility: A Case for GIs. Online J Rural Nurs Health Care. 2009; 9 (1): 46-55.

6. Andersen R, Aday A. A Theoretical Framework for the Study of Access to Medical Care. Health Serv Res; 1974; 9 (3): 208-22.

7. Organización de Naciones Unidas (ONU). Realizing the Future We Want for All. Report to the Secretary-General. Nueva York; 2012.

8. Organización Mundial de la Salud (oms). Informe sobre la salud del mundo 2013. Investigaciones para una cobertura sanitaria universal; 2013[informe en Internet] [acceso: 25 de julio del 2014]. Disponible en: http://www.who.int/whr/es/
9. Grupo de Economía de la Salud (GES). Implicaciones económicas y fiscales de la cobertura universal en salud. Observatorio de la Seguridad Social. 2012; 25.

10. Grupo de Economía de la Salud (GES). Limitaciones y derechos sobre la atención en salud de los colombianos. Observatorio de la Seguridad Social. 2008; 18.

11. Ruiz F, Uprimny M. Sistema de salud y aseguramiento social entre la reforma estructural y el ajuste regulatorio. Bogotá: Ecoe Ediciones; 2012.

12. Uprimny R, Durán J. Equidad y protección social del derecho a la salud en Colombia. Cepal - Serie Políticas Sociales. 2014; (197).

13. Vargas J, Molina G. Acceso a los servicios de salud en seis ciudades de Colombia: limitaciones y consecuencias. Rev. Salud Pública. 2009; 27 (2): 121-30.

14. Zambrano A, Ramírez M, Yepes FJ, Guerra JA, Rivera D. What Do Living Standard Surveys Show about the Health System in Colombia? Cad. Saúde Pública.2008 ene; 24 (1): 122-30.

15. Ruiz F. Bases técnicas de la reforma. Observatorio de la Seguridad Social. 2013; 26: 3-8.

16. Hirmas Adauy M, Poffald Angulo L, Jasmen Sepúlveda AM, Aguilera Sanhueza X, Delgado Becerra I, Vega Morales J. Health Care Access Barriers and Facilitators: A Qualitative Systematic Review. Rev Panam Salud Pública. 2013 mar; 33(3): 223-9.

17. Vargas-Lorenzo I, Vázquez-Navarrete ML, Mogollón-Pérez AS. Acceso a la atención en salud en Colombia. Rev. Salud Pública. 2010; 12(5): 701-712.

18. Restrepo J, Echeverri E, Vásquez J, Rodríguez S. El seguro subsidiado y el acceso a los servicios de salud: Teoría, contexto colombiano y experiencia en Antioquia. Medellín: Centro de Investigaciones Económicas, Universidad de Antioquia; 2006.

19. Rodríguez S. Roldán P. Estimación de los determinantes del acceso a los servicios de salud en la región caribe. Rev Economía del Caribe. 2008; (2).

20. Arboleda W, Murillo R, Piñeros M, Perry F, Díaz S, Salguero E. Cobertura de examen clínico y mamografía de tamización para cáncer de mama en mujeres bogotanas. Rev Col Cancerol. 2009; 13(2): 69-76.

21. Wiesner-Ceballos C, Vejarano-Velandia M, Caicedo-Meira JC, Tovar-Murillo SL, CendalesDuarte R. La citología de cuello uterino en Soacha, Colombia: Representaciones sociales, barreras y motivaciones. Rev. Salud Pública. 2006; 8 (3): 185-196.

22. Moreno G, Monsalve B, Tabima G, Escobar J. Apreciaciones de la población en condición de desplazamiento forzado sobre los servicios de salud en algunos municipios de Colombia. Rev Salud Pública 2009; 27 (2): 131-41.

23. Abadía CE, Oviedo DG. Bureaucratic Itineraries in Colombia. A Theoretical and Methodological Tool to Assess Managed-Care Health Care Systems. Soc Sci Med. 2009 mar; 68 (6): 1153-60.

24. Vargas I, Unger J-P, Mogollón-Pérez AS, Vázquez ML. Effects of Managed Care Mechanisms on Access to Healthcare: Results from a Qualitative 
Study in Colombia. The Int J Health Plan M. 2013; 28 (1): e13-e33.

25. García-Subirats I, Vargas I, Mogollón-Pérez AS, De Paepe P, Ferreira da Silva MR, Unger JP, Vázquez ML. Barriers in Access to Healthcare in Countries with Different Health Systems. A Cross-Sectional Study in Municipalities of Central Colombia and North-Eastern Brazil. Soc Sci Med. 2014 abr; 106: 204-13.

26. Secretaría de Salud de Medellín (ssm). Plan de salud municipal 2012-2015: "Medellín, Ciudad Saludable”. Alcaldía de Medellín. Rev. Salud Pública de Medellín. 2012 enero-julio; 5 (1): 13-123.
27. Valencia-Sierra ML, González-Echeverri, AgudeloVanegas NA, Acevedo-Arenas L, Vallejo Zapata IC. Acceso a los servicios de urgencias en Medellín. Rev. Salud Pública. 2007; 9(4): 529-40.

28. Restrepo JH, Mejía A, Valencia M, Tamayo L, Salas W. Accesibilidad a la citología cervical en Medellín, Colombia en 2006. Rev Esp Salud Pública. 2007; 81: 657-66.

29. Echeverry M. Reforma a la salud y reconfiguración de la trayectoria de acceso a los servicios de salud desde la experiencia de los usuarios en Medellín, Colombia. Rev Gerenc Polit Salud. 2011; 10 (20): 97-109.

30. Donabedian A. Aspects of Medical Care Administration. Cambridge: Harvard University Press; 1973. 